\title{
Novel mechanism of intra-renal angiotensin II-induced sodium/proton exchanger 3 expression by losartan in spontaneously hypertensive rats
}

\author{
XIAOQIN FAN ${ }^{1}$, KAISHAN LIU ${ }^{2}$, WEI CUI ${ }^{3}$, JIONGMEI HUANG ${ }^{4}$, WEINA WANG ${ }^{5}$ and YUAN GAO ${ }^{4}$ \\ ${ }^{1}$ Institution of Life and Health Engineering, Jinan University, Guangzhou, Guangdong 510632; ${ }^{2}$ Department of Laboratory, \\ The Border Armed Police Central Hospital of Guangdong Province, Shenzhen, Guangdong 518023; ${ }^{3}$ Department of \\ Rehabilitation, The First People's Hospital of Foshan, Foshan, Guangdong 528000; ${ }^{4}$ Department of Physiology, \\ Zunyi Medical College, Zhuhai Campus, Zhuhai, Guangdong 519041; ${ }^{5}$ Department of Physiology, \\ Nanyang Medical College, Nanyang, Henan 473000, P.R. China
}

Received November 19, 2013; Accepted June 20, 2014

DOI: $10.3892 / \mathrm{mmr} .2014 .2492$

\begin{abstract}
The present study aimed to investigate the molecular pharmacodynamic mechanisms of losartan used in the treatment of hypertension. A total of 12 spontaneously hypertensive rats (SHR) were divided randomly into an SHR group treated with saline and LOS group treated with losartan. Six Wistar-kyoto rats (WKY) were enrolled as the WKY group with saline in the study. The LOS group received $30 \mathrm{mg} / \mathrm{kg} /$ day losartan by intragastric injection, while the SHR and WKY were fed the same volume of saline. The dosage was modulated according to the weekly weight. Changes in blood pressure were measured by the indirect tail cuff method. Angiotensin (Ang) II production in the plasma and renal tissue was measured by an immunoradiometric method. $\mathrm{Na}^{+} / \mathrm{H}^{+}$exchanger (NHE)3 and serum and glucocorticoid-inducible kinase (SGK)1 were assessed by quantitative polymerase chain reaction (qPCR) and western blot analysis. When compared with the WKY group, the blood pressure of the SHR and LOS groups were higher prior to treatment with losartan. Following two weeks, blood pressure was reduced and the trend continued to decrease over the following six weeks. The plasma and renal tissue levels of Ang II in the SHR and LOS groups were significantly higher than those in the WKY group. NHE3 and SGK1 were increased at the mRNA and protein level in the SHR group, and losartan reduced the expres-
\end{abstract}

Correspondence to: Professor Yuan Gao, Department of Physiology, Zunyi Medical College, Zhuhai Campus, Room 212, Administration Building, Jinhai Avenue, Jinwan, Zhuhai, Guangdong 519041, P.R. China

E-mail: gy60211@aliyun.com

Abbreviations: SHR, spontaneously hypertensive rats; WKY, Wistar kyoto rats; 1OS, Losartan; Ang II, angiotensin II; BP, blood pressure; RAS, renin angiotensin system; NHE3, sodium/proton exchanger 3; SGK1, serum and glucocorticoid inducible kinase 1

Key words: losartan, hypertension, angiotensin, sodium/proton exchanger 3 sion of both of them. The results suggested that in hypertensive rats, the circular and tissue renin angiotensin systems were activated, and the increased Ang II stimulated the expression of NHE3 and SGK1, which was reduced by losartan. Therefore, the effects of losartan in hypertension may be associated with the Ang II-SGK1-NHE3 of intra-renal tissue.

\section{Introduction}

Hypertension is recognized as one of the most frequent health concerns for the human population (1). There are numerous signaling pathways and feedback mechanisms that contribute to the process of hypertension and its associated diseases, including renal dysfunction and diabetes. The renin angiotensin system (RAS) has been demonstrated to be the main mediator in the pathogenesis of hypertension. RAS acts not only at a circulatory level but also at the tissue level, including the heart, kidney and brain, which are all of marked significance for the development of hypertension (2-4).

The principal peptide of the RAS is angiotensin II (Ang II), which acts by binding to one of the two major Ang II receptors AT1 and AT2 (5). In the kidney, Ang II, through the Ang II receptor type 1 (AT1 receptor), increases sodium retention and pressure within the glomeruli, which accelerates the progression of hypertension. The increased sodium retention by the proximal tubule is mediated by the $\mathrm{Na}^{+} / \mathrm{H}^{+}$exchanger (NHE) (6). NHE is a ubiquitous transport system that is involved in the regulation of intracellular $\mathrm{pH}$, cell volume, cell growth and proliferation, and transepithelial absorption of $\mathrm{Na}^{+}, \mathrm{Cl}^{-}$and $\mathrm{HCO}_{3}^{-}$(7). Several isoforms of NHE have been identified in the kidney (8). With mRNA probes, NHE1 expression was identified at the basolateral membrane of renal epithelial cells. NHE-3, a tissue-specific isoform, is localized on the thick ascending limbs of the loop of Henle, the luminal membrane of proximal tubules and certain long thin descending limbs, as detected by low-stringency screening of cDNA libraries (9). NHE3 regulates bicarbonate absorption, salt, volume homeostasis and tubular protein expression. Renal NHE, particularly the NHE3 isoform, are essential for the maintenance of acid-base homeostasis. Both the expres- 
sion and NHE activity are subject to complex regulation. In diabetic animal models, NHE3 expression is highly stimulated in the early phase, which is characterized by increased glomerular filtration rate. In conclusion, NHE has an important role in renal physiology and response to diabetes (10). Serum and glucocorticoid-inducible kinase 1 (SGK1) is expressed following exposure to a variety of hormones in kidney. SGK1 enhances the activity of a variety of carriers (NHE3, NHE1, SGLT1) and ion channels (renal outer medullary potassium channel, potassium voltage-gated channel subfamily E member 1/subfamily Q member 1, metabotropic glutamate receptor 6 and cystic fibrosis transmembrane conductance regulator). SGK1 contributes to $\mathrm{Na}^{+}$retention and $\mathrm{K}^{+}$elimination in the kidney. Therefore, SGK1 may participate in the pathogenesis of hypertension $(11,12)$.

Clinically, blockade of the RAS is the prominent therapeutic strategy for the treatment of hypertension. The administration of angiotensin-converting enzyme inhibitors and Ang II receptor blockers are widely used in the treatment of hypertension (13). Losartan is a selective and competitive antagonist for the AT1 receptor. Currently, losartan is a highly popular antihypertensive agent with well-recognized clinical efficacy supported by large-scale clinical studies. However, the molecular mechanisms underlying the beneficial effects of losartan remain elusive.

The present study examined the expression of renal sodium/proton exchangers, particularly NHE3, affected by activated the RAS of renal tissue, in spontaneously hypertensive rats and aimed to elucidate the molecular pharmacodynamic mechanism of losartan.

\section{Materials and methods}

Subjects. A total of 12 spontaneously hypertensive rats and six Wistar-kyoto rats (WKY; male or female) aged 10 weeks, weighing $150 \mathrm{~g}$ from Vital River Laboratories Co., Ltd. (Beijing, China) were enrolled in the study. The spontaneously hypertensive rats were randomly divided into two groups: The SHR group (saline, six animals); the LOS group (losartan-treated, six animals). The rats were housed under conditions of constant temperature $\left(24^{\circ} \mathrm{C}\right)$ and humidity $(60 \%)$, exposed to a 12-h light/dark cycle, and provided tap water to drink, as well as SPF level standard rat feed. The experimental design was approved by the Institutional Ethics Committee of the Institute of Zunyi Medical College (Zhuhai, Guangdong, China).

Reagents. Losartan was purchased from Merck USA (Whitehouse Station, NJ, USA). Monoclonal antibody NHE3, monoclonal antibody SGK1 and immunohistochemical staining avidin-biotin-peroxidase complex (ABC) kit were purchased Santa Cruz Biotechnology, Inc. (Santa Cruz, CA, USA). The First Strand cDNA Synthesis kit and 2x AllinOneTM Q-PCR Mix were purchased from GeneCopoeia (Guangzhou, China), and the Angiotensin II radioimmunoassay kit was purchased from HTA Co. Ltd. (Beijing, China).

Blood pressure $(\mathrm{BP})$ measurement. The $\mathrm{BP}$ was measured by the indirect tail cuff method, using an RM-6240 multi-channel physiological signal acquisition and processing system (Chengdu Instrument Factory, Sichuan, China). The measurements were performed while the rats were maintained in a temperature-regulated holder. The mean of three consecutive readings was used for BP determination.

Immunoradiometric detected of Ang II. An immunoradiometric method was utilized for the detection of plasma and renal Ang II by a radioimmunoassay kit according to the manufacturer's instructions (HTA Co. Ltd., Beijing, China).

Immunohistochemical demonstration of NHE3 in kidney tissue. The kidney tissues were fixed for $12-24 \mathrm{~h}$ in $4 \%$ paraformaldehyde and following dehydration, were embedded in paraffin. The tissues were cut into $5-\mu \mathrm{m}$ thick sections, which were then mounted onto glass slides, and following deparaffinization and hydration, were immunostained according to the manufacturer's instructions. The sections were sequentially exposed to normal goat serum for $1 \mathrm{~h}$ and then to NHE3 antibody at a final dilution of 1:200 overnight at $4^{\circ} \mathrm{C}$. Next, samples were labeled with the $\mathrm{ABC}$ complex diluted 1:200 for $30 \mathrm{~min}$. The peroxidase label was revealed by reaction with stable diaminobenzidine. Photomicrographs were obtained with an Axiovert 200 inverted fluorescence microscope (Zeiss, Oberkochen, Germany). Six images of six fields of the histological sections were acquired. The samples were considered positive when $>30 \%$ of the tissue components were immunohistochemically stained brown-yellow in the appropriate cellular compartment.

Reverse transcription-polymerase chain reaction. Total RNA was isolated from renal cortex using TRIzol (Invitrogen Life Technologies, Carlsbad, CA, USA) and the DNA was removed by the recombinant DNaseI. For qPCR, cDNA was prepared from $1 \mu \mathrm{g}$ total RNA, using reverse transcriptase from the First Strand cDNA Synthesis kit in accordance with the manufacturer's instructions. All PCRs were conducted using the 2x AllinOneTM Q-PCR Mix, and cycling parameters $95^{\circ} \mathrm{C}, 15 \mathrm{sec} ; 60^{\circ} \mathrm{C}, 15 \mathrm{sec}$; and $72^{\circ} \mathrm{C}, 20 \mathrm{sec}$ for 39 cycles, with primers designed against the following mouse sequences:

\section{GAPDH: (forward, 5'-GGACCAGGTTGTCTCCTGTG-3' reverse, 5'-TGTAGGCCATGAGGTCCAC-3') \\ NHE3: (forward, 5'-AGGACAAATTGGACACAATTACC-3' reverse, 5'-GCTCATGGAAAACATTCAGGA-3') \\ SGK1: (forward, 5'-CTGTTCTACCATCTCCAGAG-3' reverse, 5'-CCGTAGAGCATCTCATACAG-3')}

Quantification of NHE3 and SGK1 protein by western blot analysis. The whole tissue lysates were separated on an $8 \%$ polyacrylamide gel. The proteins were transferred to a polyvinylidene difluoride membrane. The membranes were blocked with $10 \%$ skimmed milk in Tris-buffered saline-Tween-20 (TBST) for $1 \mathrm{~h}$, and then probed with primary antibodies diluted in $5 \%$ milk in TBST overnight at $4^{\circ} \mathrm{C}$. The membranes were washed three times with TBST and then incubated with secondary antibodies at room temperature for $1 \mathrm{~h}$ and developed using BeyoECL Plus kit (Beyotime Institute of Biotechnology, Jiangsu, China).

\section{Results}

Effect of losartan on the BP. The baseline BP in the spontaneously hypertensive rats was significantly higher than that in the 


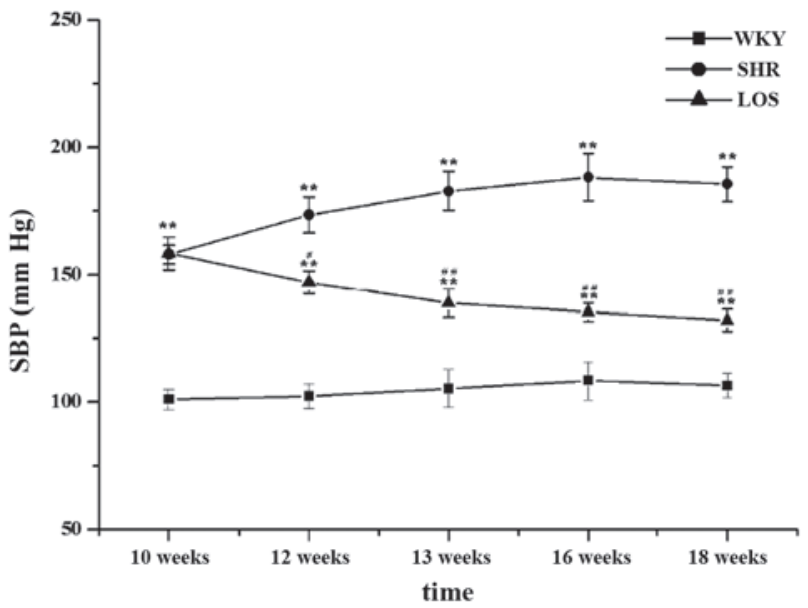

Figure 1. Effect of losartan on the systolic blood pressure in SHR. Baseline blood pressure in the SHR and LOS groups was higher than the WKY group at 10 weeks prior to the experiment $(" \mathrm{P}<0.01)$. Following losartan treatment for two weeks the SBP was significantly attenuated compared with that in the SHR group $\left(" \mathrm{P}<0.05,{ }^{\# \prime} \mathrm{P}<0.01\right)$. LOS group, losartan-treated rats; WKY, Wistar-kyoto rats; SHR, spontaneously hypertensive rats; SBP, systolic blood pressure.
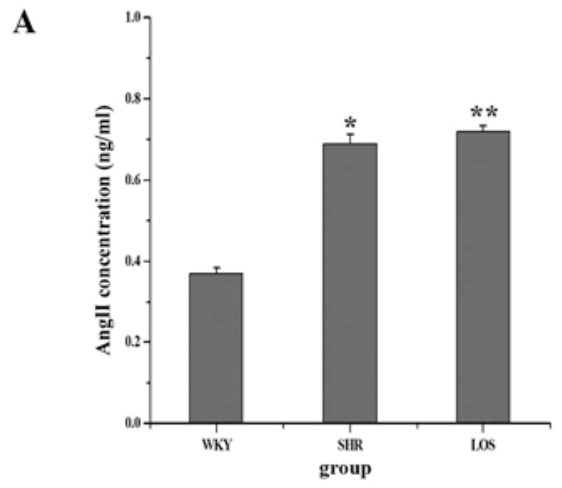

B

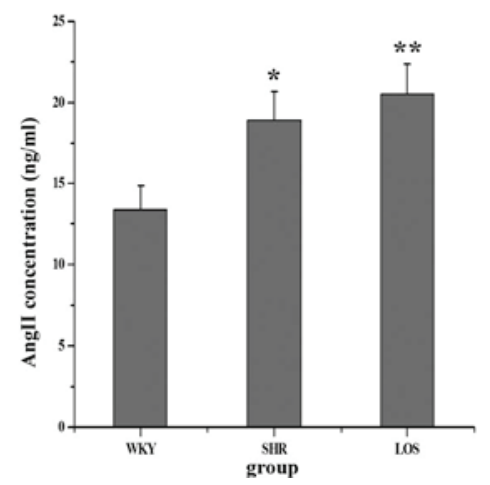

Figure 2. Effect of losartan on Ang II in the blood plasma and renal tissue in the SHR group. The Ang II levels in the (A) blood plasma and (B) renal tissue were activated in the SHR group compared with those in the WKY group. Following losartan treatment the levels of Ang II remained higher than those in the WKY group, but showed no difference compared with the SHR group. Values are presented as the mean \pm standard error of the mean of each group $\left({ }^{*} \mathrm{P}<0.05\right.$ and ${ }^{* * *} \mathrm{P}<0.01$ vs. the WKY group). LOS group, losartan-treated rats; WKY, Wistar-kyoto rats; Ang II, angiotensin II; SHR, spontaneously hypertensive rats.

WKY rats at ten weeks (Fig. 1). Treatment with losartan attenuated BP compared with the SHR group following two weeks, and the tendency of the BP remained attenuate with continuous losartan treatment in a time-dependent manner following eight
A

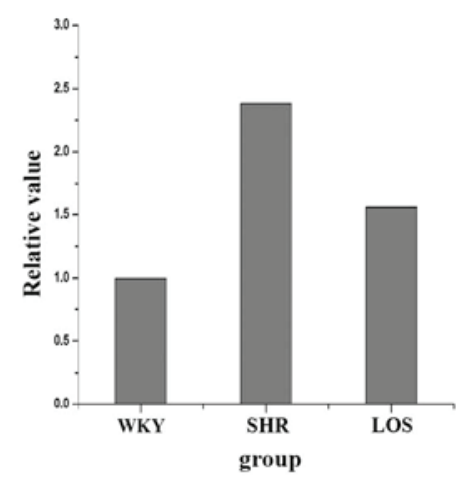

B

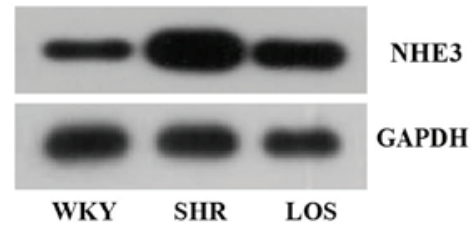

C

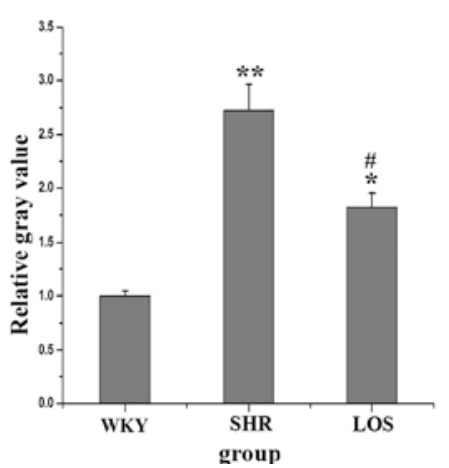

Figure 3. Effect of losartan on NHE3 mRNA and protein expression. (A) Quantitative polymerase chain reaction demonstrating an increase in NHE3 mRNA expression in the SHR group compared with that in the WKY group, while losartan attenuated the mRNA expression of NHE3. (B) Western blot analysis demonstrating an increase in NHE3 protein expression in the SHR group compared the WKY group but losartan attenuated the protein expression of NHE3. (C) Relative gray values of the western blot analysis of B. Values are presented as the mean \pm standard error of the mean of each group. Compared with the WKY group, the SHR and LOS groups were significantly higher $\left({ }^{*} \mathrm{P}<0.05\right.$; $\left.{ }^{* *} \mathrm{P}<0.01\right)$. Compared with the SHR group, the LOS group was significantly lower $\left({ }^{*} \mathrm{P}<0.05\right)$. LOS group, losartan-treated rats; WKY, Wistar-kyoto rats; SHR, spontaneously hypertensive rats; NHE3, $\mathrm{Na}^{+} / \mathrm{H}^{+}$exchanger 3 .

weeks. There was a significant difference between the SHR and the LOS groups, but the blood pressure was still higher in the WKY group.

Effect of losartan on Ang II in the plasma and renal tissue. In the present study, the concentration of Ang II in plasma and renal tissue was examined in order to confirm that in hypertension, both at circulatory and tissue levels, the RAS was activated. As the results demonstrate in Fig. 2, the levels of Ang II in the SHR and LOS groups were significantly higher than those in the WKY group, not only in plasma (Fig. 2A) but also in renal tissues (Fig. 2B). However, following losartan treatment for eight weeks, the concentration of Ang II in plasma and renal tissue exhibited no significant difference between the SHR and LOS groups, but remained markedly higher than that in the WKY group. Therefore, the RAS at the circulatory and tissue levels was activated in the 
Aa

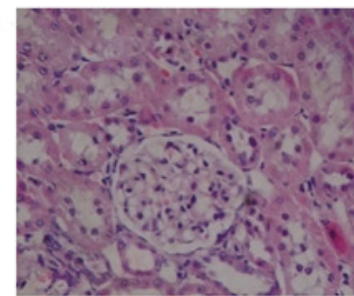

b

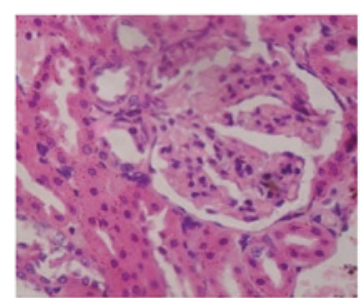

c

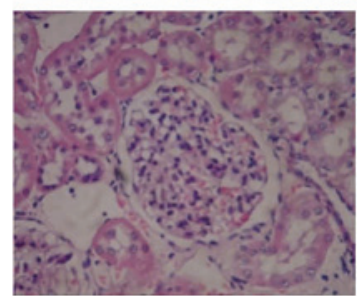

Ba

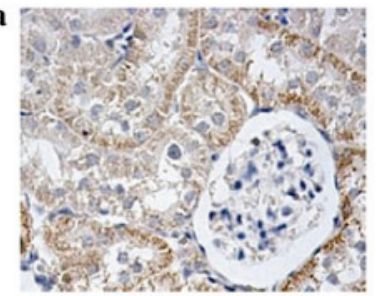

b

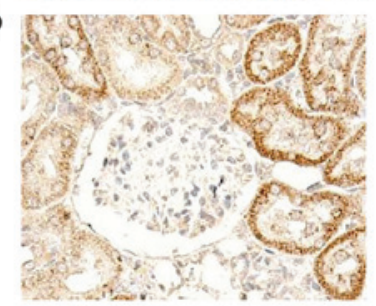

c

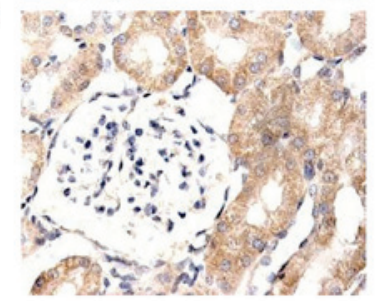

C

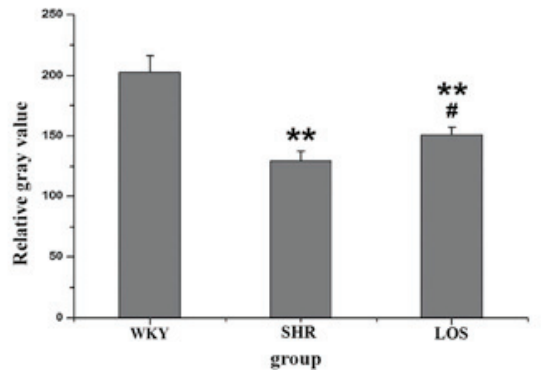

Figure 4. Effect of losartan on histological and NHE3 changes observed by $\mathrm{H} \& \mathrm{E}$ and IHC staining, respectively. (A) H\&E staining demonstrated the changes of renal tissue in the (a) WKY group, (b) SHR group and (c) LOS group. (B) IHC staining demonstrated the changes of NHE3 in renal tissue in the (a) WKY group, (b) SHR group and (c) LOS group. (C) The relative gray values of the western blot analysis results of B. Values are presented as the mean \pm standard error of the mean of each group. Compared with the WKY group, the SHR and LOS groups were significantly lower $\left({ }^{* *} \mathrm{P}<0.01\right)$. However, the LOS group was significantly increased compared with the SHR group $\left({ }^{\#} \mathrm{P}<0.05\right)$. LOS group, losartan-treated rats; WKY, Wistar-kyoto rats; SHR, spontaneously hypertensive rats; $\mathrm{SBP}$, systolic blood pressure; $\mathrm{NHE} 3, \mathrm{Na}^{+} / \mathrm{H}^{+}$ exchanger 3; IHC, immunohistochemical; H\&E, hematoxylin and eosin.

hypertension and losartan groups, while the antagonist of the AT1 receptor did not decrease the concentration of Ang II in the plasma and renal tissue.

NHE3 $m R N A$ and protein expression. Previously, studies have demonstrated that Ang II increased NHE3 in human proximal tubular cells (PTCs) $(14,15)$. To investigate the effect of Ang II on NHE3 in renal tissue of hypertension-associated renal dysfunction, the mRNA and protein expression of NHE3 was examined. The mRNA expression is revealed in Fig. 3A. In the SHR and LOS groups, the relative expression increased to 2.38 and 1.56 , respectively, as compared with that in the WKY group, and losartan was able to reduce NHE3 expression. Meanwhile, the protein expression of NHE3, as revealed in Fig. 3B, was significantly increased in the SHR group, compared with that in the WKY group. Furthermore,

A

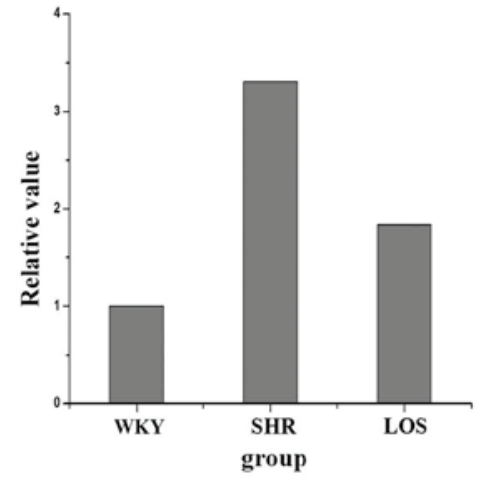

B

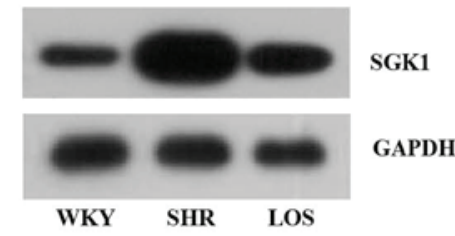

C

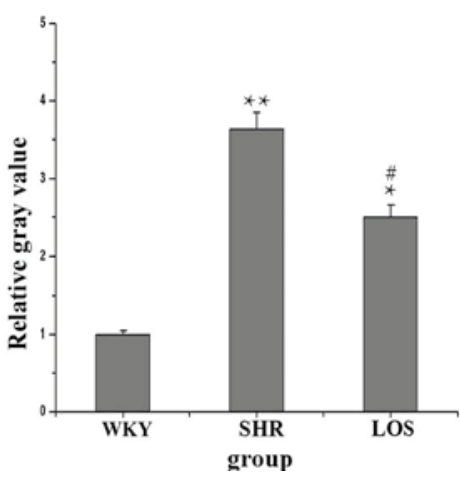

Figure 5. Effect of losartan on the SGK1 mRNA and protein expression. (A) Quantitative polymerase chain reaction demonstrating an increase in the SGK1 mRNA expression in the SHR group compared the WKY group, but losartan attenuated the mRNA expression of SGK1. (B) Western blot demonstrating an increase in the SGK1 protein expression in the SHR group compared with that in the WKY group, while losartan attenuated the protein expression of SGK1. (C) Relative gray values of western blot analysis result of B. Values are presented as the mean \pm standard error of the mean of each group. Compared with the WKY group, the SHR and LOS groups were significantly increased ( $\left.{ }^{*} \mathrm{P}<0.05 ;{ }^{* *} \mathrm{P}<0.01\right)$, whilst the LOS group was significantly reduced compared with the SHR group $\left({ }^{*} \mathrm{P}<0.05\right)$. LOS group, losartan-treated rats; WKY, Wistar-kyoto rats; SHR, spontaneously hypertensive rats; SBP, systolic blood pressure; SGK1, serum and glucocorticoid-inducible kinase 1; IHCS, immunohistochemical staining; H\&E, hematoxylin and eosin.

following eight weeks of losartan treatment, the mRNA expression levels of NHE3 were decreased.

Effect of losartan on histopathology and NHE3 expression in renal tissue. The renal tissue was stained using traditional hematoxylin and eosin methods. Fig. 4A reveals the significant morphological changes in renal tissue that were observed, including glomerular dilatation and detachment of basement membrane from glomerulus, in the SHR group as compared with the WKY group. The damage in the renal tissue was ameliorated by treatment with losartan. Representative immunohistochemistry (IHC) images of NHE3 in the renal tissue are demonstrated in Fig. 4B. NHE3 staining was detected predominantly in the PTCs in the WKY group. Intense 
staining was noted in the brush borders and thick ascending limbs of the loop of Henle, except for the PTCs in the SHR group. These effects were weakened by losartan treatment. The quantified immunostaining intensities of NHE3 are revealed in (Fig. 4C).

SGK1 mRNA and protein expression. The effect of Ang II, via the AT1 receptor, on the mRNA and protein expression of NHE3 was examined, but the mechanism underlying this effect remained elusive. Previously, Stevens et al (16) confirmed that the Ang II-induced increase in the NHE3 expression was mediated through SGK1 in human renal PTCs of diabetes mellitus. It was therefore considered that Ang II may regulate NHE3 through an SGK1-dependent mechanism in hypertension-associated renal dysfunction (17). The present study investigated the mRNA and protein expression of SGK1 in renal tissue. The data (Fig. 5) demonstrated that the mRNA expression of SGK1 in the SHR and LOS groups was increased to 3.31 and 1.84 compared with that in the WKY group. Furthermore, the protein expression in the SHR and LOS groups was higher than in the WKY group. However, it was demonstrated that losartan treatment was able to significantly decrease the expression of SGK1.

\section{Discussion}

At present, hypertension affects $\sim 1$ billion individuals worldwide, and therefore, it is highly important to investigate the molecular mechanisms underlying the pathogenesis of hypertension and the activities of anti-hypertensive drugs. Numerous different factors contribute to the development of hypertension, including the RAS, which has an important role in the regulation and progression of hypertension. The major therapeutic strategies for the clinical treatment of hypertension are angiotensin-converting enzyme inhibitors and Ang II receptor blockers. Losartan is widely used in the clinic and is a typical representative Ang II receptor blocker, but the molecular mechanism underlying its effects have remained elusive.

The rats of the SHR and WKY groups have the same genetic background and used in the present study. In the present study, it was identified that the baseline BP of spontaneously hypertensive rats was reduced significantly by losartan following administration via intragastric injection over two weeks, and the BP continued to decline after this time-point. This demonstrated that losartan was effective in reducing the hallmarks of hypertension, which is consistent with the results of other studies (18-20). Next, it was confirmed that the circulatory and tissue levels of Ang II in the SHR group were higher than those in the WKY group, while Ang II levels in the LOS group were even higher than those in the SHR group. It was considered that losartan may exert its effects by increasing Ang II compensation.

The intra-renal RAS and the effects of RAS blockade on lead-induced nephropathy in hypertension were also examined. It has been demonstrated in PTCs of diabetes that Ang II alone at renal concentrations stimulates NHE3 mRNA and protein expression, which is mediated by SGK1, as reflected by increased $\mathrm{Na}^{+}$reabsorption $(16,21,22)$. This reveals an important role for the intra-renal Ang II-NHE3-SGK1 pathway in regulating $\mathrm{Na}^{+}$uptake. Therefore, whether blocking of the intra-renal Ang II by losartan in hypertension may also affect this pathway was of particular interest in the present study.

Therefore, using qPCR and western blot analysis, it was possible to detect the mRNA and protein expression of NHE3 and SGK1 in renal tissue and demonstrate that both of them were upregulated in the SHR group compared with the WKY group. It was also identified that losartan reduced the mRNA and protein expression of NHE3 and SGK1. Furthermore, the histopathology and IHC determination of NHE3 in the renal tissue revealed that hypertension-associated nephropathy was reduced by losartan in the SHR group. In conclusion, in the present study revealed that the intra-renal RAS and Ang II-NHE3-SGK1 pathway were activated in renal tissue under hypertension, and that losartan reduced the hypertensive effect in the SHR group effectively via this pathway. The present study provided evidence for a new pharmacodynamic mechanism of action of losartan in the treatment of hypertension.

\section{Acknowledgements}

This study was funded by grants from the Natural Science Foundation (grant no. 31160214).

\section{References}

1. Gu D, Reynolds K, Wu X, et al: Prevalence, awareness, treatment, and control of hypertension in China. Hypertension 40: 920-927, 2002.

2. Griendling KK, Murphy T and Alexander RW: Molecular biology of the renin-angiotensin system. Circulation 87: 1816-1828, 1993

3. Peach MJ: Renin-angiotensin system: biochemistry and mechanisms of action. Physiol Rev 57: 313-370, 1977.

4. Kobori H, Nangaku M, Navar LG and Nishiyama A: The intrarenal renin-angiotensin system: from physiology to the pathobiology of hypertension and kidney disease. Pharmacol Rev 59: 251-287, 2007.

5. Paul M, Mehr AP and Kreutz R: Physiology of local renin-angiotensin systems. Physiological Reviews 86: 747-803, 2006.

6. Biemesderfer D, Pizzonia J, Abu-Alfa A, et al: NHE3: a Na ${ }^{+} / \mathrm{H}^{+}$ exchanger isoform of renal brush border. Am J Physiol 265: F736-F742, 1993.

7. Malo ME and Fliegel L: Physiological role and regulation of the $\mathrm{Na}^{+} / \mathrm{H}^{+}$exchanger. Can J Physiol Pharmacol 84: 1081-1095, 2006.

8. Orlowski $\mathrm{J}$ and Grinstein S: Diversity of the mammalian sodium/proton exchanger SLC9 gene family. Pfluegers Archiv 447: 549-565, 2004

9. Amemiya M, Loffing J, Lötscher M, et al: Expression of NHE-3 in the apical membrane of rat renal proximal tubule and thick ascending limb. Kidney Int 48: 1206-1215, 1995.

10. Khan I, Batinic-Haberle I and Benov LT: Effect of potent redox-modulating manganese porphyrin, MnTM-2-PyP, on the $\mathrm{Na}^{+} / \mathrm{H}^{+}$exchangers NHE- 1 and NHE-3 in the diabetic rat. Redox Rep 14: 236-242, 2009.

11. Pao AC: SGK regulation of renal sodium transport. Curr Opin Nephrol Hypertens 21: 534-540, 2012.

12. Lang F, Huang DY and Vallon V: SGK, renal function and hypertension. J Nephrol 23 (Suppl 16): S124-S129, 2010.

13. Smith DH: Comparison of angiotensin II type 1 receptor antagonists in the treatment of essential hypertension. Drugs 68: 1207-1225, 2008.

14. Xu L, Dixit MP, Nullmeyer KD, et al: Regulation of $\mathrm{Na}^{+} / \mathrm{H}^{+}$ exchanger-NHE3 by angiotensin-II in OKP cells. Biochimica et Biochim Biophys Acta 1758: 519-526, 2006.

15. Moe OW: Acute regulation of proximal tubule apical membrane $\mathrm{Na} / \mathrm{H}$ exchanger NHE-3: role of phosphorylation, protein trafficking, and regulatory factors. J Am Soc Nephrol 10: 2412-2425, 1999. 
16. Stevens VA, Saad S, Poronnik P, et al: The role of SGK-1 in angiotensin II-mediated sodium reabsorption in human proximal tubular cells. Nephrol Dial Transplant 23: 1834-1843, 2008.

17. Wang D, Sun H, Lang F and Yun CC: Activation of NHE3 by dexamethasone requires phosphorylation of NHE3 at Ser663 by SGK1. Am J Physiol Cell Physiol 289: C802-C810, 2005.

18. Lu D, Raizada MK, Iyer S, et al: Losartan versus gene therapy chronic control of high blood pressure in spontaneously hypertensive rats. Hypertension 30: 363-370, 1997.

19. Baumann M, Janssen B, Rob Hermans J, et al: Renal medullary effects of transient prehypertensive treatment in young spontaneously hypertensive rats. Acta Physiologica (Oxf.) 196: 231-237, 2009.
20. Koprdova R, Cebova M and Kristek F: Long-term effect of losartan administration on blood pressure, heart and structure of coronary artery of young spontaneously hypertensive rats. Physiol Res 58: 327-325, 2009.

21. Kaunisto KM and Rajaniemi HJ: Expression and localization of the $\mathrm{Na}^{+} / \mathrm{H}^{+}$exchanger isoform NHE3 in the rat efferent ducts. J Androl 23: 237-241, 2002.

22. Karim Z, Gérard B, Bakouh N, et al: NHERF1 mutations and responsiveness of renal parathyroid hormone. N Engl J Med 359: 1128-1135, 2008. 